\title{
ISHIKAWA ITERATION PROCESS WITH ERRORS FOR NONEXPANSIVE MAPPINGS
}

\author{
JIALIN HUANG
}

(Received 16 June 2000)

\begin{abstract}
We study the construction and the convergence of the Ishikawa iterative process with errors for nonexpansive mappings in uniformly convex Banach spaces. Some recent corresponding results are generalized.
\end{abstract}

2000 Mathematics Subject Classification. 47H10, 40A05.

1. Introduction. Let $C$ be a closed convex subset of a Banach space $X$ and $T: C \rightarrow C$ be a nonexpansive mapping (i.e., $\|T x-T y\| \leq\|x-y\|$ for all $x, y$ in $C$ ). Recently, Deng and Li [1] introduced an Ishikawa iteration sequence with errors as follows: for any given $x_{0} \in C$

$$
\begin{gathered}
x_{n+1}=\alpha_{n} x_{n}+\beta_{n} T y_{n}+\gamma_{n} u_{n}, \\
y_{n}=\hat{\alpha}_{n} x_{n}+\hat{\beta}_{n} T x_{n}+\hat{\gamma}_{n} v_{n}, \quad n \geq 0 .
\end{gathered}
$$

Here $\left\{u_{n}\right\}$ and $\left\{v_{n}\right\}$ are two bounded sequences in $C$, and $\left\{\alpha_{n}\right\},\left\{\beta_{n}\right\},\left\{\gamma_{n}\right\},\left\{\hat{\alpha}_{n}\right\}$, $\left\{\hat{\beta}_{n}\right\}$, and $\left\{\hat{\gamma}_{n}\right\}$ are six sequences in $[0,1]$ satisfying the conditions

$$
\alpha_{n}+\beta_{n}+\gamma_{n}=\hat{\alpha}_{n}+\hat{\beta}_{n}+\hat{\gamma}_{n}=1 \quad \forall n \geq 0 .
$$

REMARK 1.1. Note that the Ishikawa iteration processes [2] is a special case of the Ishikawa iteration processes with errors.

Deng and Li [1] obtained the following result. Let $C$ be a closed convex subset of a uniformly convex Banach space $X$. If for any initial guess $x_{0} \in C,\left\{x_{n}\right\}$ defined by (1.1), with the restrictions that $\sum_{n=0}^{\infty} \alpha_{n} \beta_{n}=\infty, \sum_{n=0}^{\infty} \alpha_{n} \beta_{n} \hat{\beta}_{n}<\infty, \sum_{n=0}^{\infty} \gamma_{n}<\infty$, and $\sum_{n=0}^{\infty} \hat{\gamma}_{n}<\infty$, then $\lim _{n \rightarrow \infty}\left\|x_{n}-T x_{n}\right\|=0$. So Deng and Li extended the result of Tan and $\mathrm{Xu}[6]$.

In this paper, we first extend and unify [1, Theorem 1] and [6, Lemma 3]. Then, we generalize [1, Theorems 2, 3, and 4] and [6, Theorems 1, 2, and 3].

\section{Lemmas}

LEMMA 2.1 (see [6]). Suppose that $\left\{a_{n}\right\}$ and $\left\{b_{n}\right\}$ are two sequences of nonnegative numbers such that $a_{n+1} \leq a_{n}+b_{n}$ for all $n \geq 1$. If $\sum_{n=1}^{\infty} b_{n}$ converges, then $\lim _{n \rightarrow \infty} a_{n}$ exists. 
LEMmA 2.2 (see [1]). Let $C$ be a closed convex subset of a Banach space $X, T: C \rightarrow C$ a nonexpansive mapping. Then for any initial guess $x_{0}$ in $C,\left\{x_{n}\right\}$ defined by (1.1),

$$
\left\|x_{n+1}-p\right\| \leq\left\|x_{n}-p\right\|+\gamma_{n}\left\|u_{n}-p\right\|+\beta_{n} \hat{\gamma}_{n}\left\|v_{n}-p\right\|
$$

for all $n \geq 1$ and for all $p \in F(T)$, where $F(T)$ denotes the set of fixed point of $T$.

REMARK 2.3. Since the sequences $\left\{u_{n}\right\}$ and $\left\{v_{n}\right\}$ are bounded, so the sequences $\left\{\left\|u_{n}-p\right\|\right\}$ and $\left\{\left\|v_{n}-p\right\|\right\}$ are bounded too, then $\lim _{n \rightarrow \infty}\left\|x_{n}-p\right\|$ exists by Lemma 2.1.

LEMMA 2.4 (see [7]). Let $C$ be a bounded closed convex subset of a uniformly convex Banach space $X$. Suppose that $T: C \rightarrow C$ is a nonexpansive mapping. If $y_{n} \rightarrow y$ weakly $\left(y_{n}, y \in C, n=1,2, \ldots\right)$, then there exists a strictly increasing convex function $g: \mathbb{R}^{+} \rightarrow \mathbb{R}^{+}$with $g(0)=0$ such that

$$
g(\|y-T y\|) \leq \liminf _{n \rightarrow \infty}\left\|y_{n}-T y_{n}\right\|
$$

\section{Main results}

THEOREM 3.1. Let $C$ be a closed convex subset of a uniformly convex Banach space $X, T: C \rightarrow C$ a nonexpansive mapping with a fixed point. If for any initial guess $x_{0}$ in $C$, $\left\{x_{n}\right\}$ defined by (1.1), with the restrictions that $\sum_{n=0}^{\infty} \gamma_{n}<\infty, \sum_{n=0}^{\infty} \hat{\gamma}_{n}<\infty$, and there exists a subsequence $\left\{n_{k}\right\}$ of $\{n\}$ such that $\sum_{k=0}^{\infty} \alpha_{n_{k}} \beta_{n_{k}}=\infty, \sum_{k=0}^{\infty} \alpha_{n_{k}} \beta_{n_{k}} \hat{\beta}_{n_{k}}<\infty$. Then $\liminf _{n \rightarrow \infty}\left\|x_{n}-T x_{n}\right\|=0$.

Proof. Since $T$ has a fixed point, and by Lemma 2.2, we may set

$$
M=\sup _{n \geq 0}\left\{\left\|T x_{n}-u_{n}\right\|,\left\|x_{n}-u_{n}\right\|,\left\|T y_{n}-v_{n}\right\|,\left\|y_{n}-u_{n}\right\|,\left\|x_{n}-v_{n}\right\|\right\}
$$

If $\liminf _{n \rightarrow \infty}\left\|x_{n}-T x_{n}\right\|>0$, we may assume that $\liminf _{n \rightarrow \infty}\left\|x_{n}-p\right\|>0$, where $p \in F(T)$. Since $\left\|T y_{n}-p\right\| \leq\left\|x_{n}-p\right\|+\hat{\gamma}_{n} M$, we obtain

$$
\begin{aligned}
\left\|x_{n+1}-p\right\| & \leq\left\|\alpha_{n}\left(x_{n}-p\right)+\beta_{n}\left(T y_{n}-p\right)\right\|+\gamma_{n} M \\
& =\left(\alpha_{n}+\beta_{n}\right)\left\|\frac{\alpha_{n}}{\alpha_{n}+\beta_{n}}\left(x_{n}-p\right)+\frac{\beta_{n}}{\alpha_{n}+\beta_{n}}\left(T y_{n}-p\right)\right\|+\gamma_{n} M \\
& \leq\left[1-2 \frac{\alpha_{n} \beta_{n}}{\left(\alpha_{n}+\beta_{n}\right)^{2}} \delta_{X}\left(\frac{\left\|x_{n}-T y_{n}\right\|}{\left\|x_{n}-p\right\|+\hat{\gamma}_{n} M}\right)\right]\left(\left\|x_{n}-p\right\|+\hat{\gamma}_{n} M\right)+\gamma_{n} M \\
& \leq\left[1-2 \alpha_{n} \beta_{n} \delta_{X}\left(\frac{\left\|x_{n}-T y_{n}\right\|}{\left\|x_{n}-p\right\|+\hat{\gamma}_{n} M}\right)\right]\left\|x_{n}-p\right\|+\left(\hat{\gamma}_{n}+\gamma_{n}\right) M,
\end{aligned}
$$

where $\delta_{X}$ is the modulus of convexity of the uniformly convex Banach space $X$. Setting

$$
D_{n}=1-2 \alpha_{n} \beta_{n} \delta_{X}\left(\frac{\left\|x_{n}-T y_{n}\right\|}{\left\|x_{n}-p\right\|+\hat{\gamma}_{n} M}\right)
$$


Thus for all $n \geq 0,0 \leq D_{n} \leq 1$. From (3.2), for all $k \geq 0$, we have

$$
\begin{aligned}
& \left\|x_{n_{k+1}}-p\right\| \\
& \quad \leq D_{n_{k+1}-1}\left\|x_{n_{k+1}-1}-p\right\|+\left(\hat{\gamma}_{n_{k+1}-1}+\gamma_{n_{k+1}-1}\right) M \\
& \quad \leq D_{n_{k+1}-1} D_{n_{k+1}-2} \cdots D_{n_{k}+1} D_{n_{k}}\left\|x_{n_{k}}-p\right\|+\sum_{i=1}^{n_{k+1}-n_{k}}\left(\hat{\gamma}_{n_{k+1}-i}+\gamma_{n_{k+1}-i}\right) M \\
& \quad \leq D_{n_{k}}\left\|x_{n_{k}}-p\right\|+\sum_{i=1}^{n_{k+1}-n_{k}}\left(\hat{\gamma}_{n_{k+1}-i}+\gamma_{n_{k+1}-i}\right) M \\
& \quad \leq\left\|x_{n_{k}}-p\right\|\left[1-2 \alpha_{n_{k}} \beta_{n_{k}} \delta_{X}\left(\frac{\left\|x_{n_{k}}-T y_{n_{k}}\right\|}{\left\|x_{n_{k}}-p\right\|+\hat{\gamma}_{n} M}\right)\right]+\sum_{i=1}^{n_{k+1}-n_{k}}\left(\hat{\gamma}_{n_{k+1}-i}+\gamma_{n_{k+1}-i}\right) M .
\end{aligned}
$$

Thus,

$$
\begin{aligned}
& \sum_{i=0}^{k} 2 \alpha_{n_{i}} \beta_{n_{i}} \delta_{X}\left(\frac{\left\|x_{n_{i}}-T y_{n_{i}}\right\|}{\left\|x_{n_{i}}-p\right\|+\hat{\gamma}_{n_{i}} M}\right)\left\|x_{n_{i}}-p\right\| \\
& \quad \leq\left\|x_{n_{0}}-p\right\|-\left\|x_{n_{k+1}}-p\right\|+\sum_{i=0}^{n_{k+1}-1}\left(\hat{\gamma}_{i}+\gamma_{i}\right) M .
\end{aligned}
$$

It follows that

$$
\sum_{i=0}^{\infty} \alpha_{n_{i}} \beta_{n_{i}} \delta_{X}\left(\frac{\left\|x_{n_{i}}-T y_{n_{i}}\right\|}{\left\|x_{n_{i}}-p\right\|+\hat{\gamma} n_{i} M}\right)<+\infty .
$$

By condition $\sum_{i=0}^{\infty} \alpha_{n_{i}} \beta_{n_{i}} \hat{\beta}_{n_{i}}<+\infty$, we have

$$
\sum_{i=0}^{\infty} \alpha_{n_{i}} \beta_{n_{i}}\left[\delta_{X}\left(\frac{\left\|x_{n_{i}}-T y_{n_{i}}\right\|}{\left\|x_{n_{i}}-p\right\|+\hat{\gamma}_{n_{i}} M}\right)+\hat{\beta}_{n_{i}}\right]<+\infty .
$$

It follows that

$$
\liminf _{k \rightarrow \infty}\left[\delta_{X}\left(\frac{\left\|x_{n_{k}}-T y_{n_{k}}\right\|}{\left\|x_{n_{k}}-p\right\|+\hat{\gamma} n_{k} M}\right)+\hat{\beta}_{n_{k}}\right]=0
$$

since $\sum_{k=0}^{\infty} \alpha_{n_{k}} \beta_{n_{k}}=\infty$. Hence, there is a sequence $\left\{n_{k_{i}}\right\} \subset\left\{n_{k}\right\}$ such that

$$
\lim _{i \rightarrow \infty}\left\|x_{n_{k_{i}}}-T y_{n_{k_{i}}}\right\|=0, \quad \lim _{i \rightarrow \infty} \hat{\beta}_{n_{k_{i}}}=0 .
$$

On the other hand, we have

$$
\begin{aligned}
\left\|x_{n_{k_{i}}}-T x_{n_{k_{i}}}\right\| & \leq\left\|x_{n_{k_{i}}}-T y_{n_{k_{i}}}\right\|+\left\|T x_{n_{k_{i}}}-T y_{n_{k_{i}}}\right\| \\
& \leq\left\|x_{n_{k_{i}}}-T y_{n_{k_{i}}}\right\|+\hat{\beta}_{n_{k_{i}}}\left\|x_{n_{k_{i}}}-T x_{n_{k_{i}}}\right\|+\hat{\gamma}_{n_{k_{i}}} M .
\end{aligned}
$$

Setting $i \rightarrow \infty$ in (3.10), it follows from (3.9) that

$$
\lim _{i \rightarrow \infty}\left\|x_{n_{k_{i}}}-T x_{n_{k_{i}}}\right\|=0
$$


Thus,

$$
\liminf _{n \rightarrow \infty}\left\|x_{n}-T x_{n}\right\|=0
$$

This completes the proof.

Recall that a Banach space $X$ is said to satisfy Opial's condition [4] if the condition $x_{n} \rightarrow x_{0}$ weakly implies

$$
\limsup _{n \rightarrow \infty}\left\|x_{n}-x_{0}\right\|<\limsup _{n \rightarrow \infty}\left\|x_{n}-y\right\| \quad \forall y \neq x_{0} .
$$

A mapping $T: C \rightarrow C$ with a nonempty fixed points set $F(T)$ in $C$ will be said to satisfy Condition $\mathrm{A}$ in [5] if there is a nondecreasing function $f:[0, \infty) \rightarrow[0, \infty)$ with $f(0)=0, f(r)>0$ for $r \in(0, \infty)$, such that $\|x-T x\| \geq f(d(x, F(T)))$ for all $x \in C$, where $d(x, F(T))=\inf \{\|x-z\|: z \in F(T)\}$.

THEOREM 3.2. Let $C$ be a bounded closed convex subset of a uniformly convex Banach space $X$ which satisfies Opial's condition or whose norm is Fréchet differentiable. Let $T: C \rightarrow C$ a nonexpansive mapping with a fixed point, and $\left\{x_{n}\right\}$ defined by (1.1), with the restrictions that $\sum_{n=0}^{\infty} \gamma_{n}<\infty, \sum_{n=0}^{\infty} \hat{\gamma}_{n}<\infty$, and for any subsequence $\left\{n_{k}\right\}$ of $\{n\}$, $\sum_{k=0}^{\infty} \alpha_{n_{k}} \beta_{n_{k}}=\infty, \sum_{k=0}^{\infty} \alpha_{n_{k}} \beta_{n_{k}} \hat{\beta}_{n_{k}}<\infty$, converges weakly to a fixed point of $T$.

By Theorem 3.1 and Lemma 2.4, we can prove Theorem 3.2 easily. The proof is similar to that of [7, Theorem 3.1], so the details are omitted.

Let $X, C, T$, and $\left\{x_{n}\right\}$ be as in Theorem 3.1. Then we have the following theorem.

THEOREM 3.3. If the range of $C$ under $T$ is contained in a compact subset of $X$, then $\left\{x_{n}\right\}$ converges strongly to a fixed point of $T$.

THEOREM 3.4. Let $C$ be a bounded closed convex subset of a uniformly convex Banach space $X$. If $T$ satisfies Condition A, then $\left\{x_{n}\right\}$ converges strongly to a fixed point of $T$.

Proof. Since $C$ is a bounded closed convex subset of a uniformly convex Banach space $X$, then $T$ has a fixed point [3]. So $F(T)$ is nonempty. It follows from Theorem 3.1 and Condition A, that there exists a subsequence $\left\{x_{n_{k}}\right\}$ of $\left\{x_{n}\right\}$ such that $\lim _{k \rightarrow \infty} f\left(d\left(x_{n_{k}}, F(T)\right)\right)=0$, therefore we have $\lim _{k \rightarrow \infty} d\left(x_{n_{k}}, F(T)\right)=0$. So we can choose a subsequence $\left\{x_{n_{k_{i}}}\right\}$ of $\left\{x_{n_{k}}\right\}$ and some sequence $\left\{p_{i}\right\}$ in $F(T)$ such that $\left\|x_{n_{k_{i}}}-p_{i}\right\|<2^{-i}$ for all integers $k \geq 0$.

We denote $\sup _{n}\left\{\left\|u_{n}-p\right\|,\left\|v_{n}-p\right\|\right\}$ by $M$ and $\left(\gamma_{n_{k_{i}}}+\beta_{n_{k_{i}}} \hat{\gamma}_{n_{k_{i}}}\right) M$ by $\lambda_{n_{k_{i}}}$. By Lemma 2.1 we have

$$
\begin{aligned}
\left\|p_{i+1}-p_{i}\right\| & \leq\left\|x_{n_{k_{i+1}}}-p_{i+1}\right\|+\left\|x_{n_{k_{i+1}}}-p_{i}\right\| \\
& \leq 2^{-(i+1)}+\left\|x_{n_{k_{i+1}}-1}-p_{i}\right\|+\lambda_{n_{k_{i+1}}-1} \\
& \leq 2^{-(i+1)}+\left\|x_{n_{k_{i+1}}-2}-p_{i}\right\|+\lambda_{n_{k_{i+1}}-2}+\lambda_{n_{k_{i+1}}-1}
\end{aligned}
$$




$$
\begin{aligned}
& \leq 2^{-(i+1)}+\left\|x_{n_{k_{i}}}-p_{i}\right\|+\sum_{j=n_{k_{i}}}^{n_{k_{i+1}}-1} \lambda_{j} \\
& \leq 2^{-i+1}+\sum_{j=n_{k_{i}}}^{n_{k_{i+1}}-1} \lambda_{j} .
\end{aligned}
$$

It follows, from (3.14) and $\sum_{j} \lambda_{j}$ is convergent, that $\left\{p_{i}\right\}$ is a Cauchy sequence therefore converges strongly to a point $p \in F(T)$, since $F(T)$ is closed. We have seen that $\left\{x_{n_{k_{i}}}\right\}$ converges strongly to $p$, so does $\left\{x_{n}\right\}$ by the Remark 2.3. This completes the proof.

REMARK 3.5. The above three theorems generalize [6, Theorems 1, 2, and 3] and [1, Theorems 2, 3, and 4], respectively.

\section{REFERENCES}

[1] L. Deng and S. Li, Ishikawa iteration process with errors for nonexpansive mappings in uniformly convex Banach spaces, Int. J. Math. Math. Sci. 24 (2000), no. 1, 49-53. CMP 1773 969. Zbl 958.47029.

[2] S. Ishikawa, Fixed points by a new iteration method, Proc. Amer. Math. Soc. 44 (1974), 147-150. MR 49\#1243. Zbl 286.47036.

[3] W. A. Kirk, A fixed point theorem for mappings which do not increase distances, Amer. Math. Monthly 72 (1965), 1004-1006. MR 32\#6436. Zbl 141.32402.

[4] Z. Opial, Weak convergence of the sequence of successive approximations for nonexpansive mappings, Bull. Amer. Math. Soc. 73 (1967), 591-597. MR 35\#2183. Zbl 179.19902.

[5] H. F. Senter and W. G. Dotson, Jr., Approximating fixed points of nonexpansive mappings, Proc. Amer. Math. Soc. 44 (1974), 375-380. MR 49\#11333. Zbl 299.47032.

[6] K. K. Tan and H. K. Xu, Approximating fixed points of nonexpansive mappings by the Ishikawa iteration process, J. Math. Anal. Appl. 178 (1993), no. 2, 301-308. MR 94g:47076. Zbl 895.47048.

[7] L.-C. Zeng, A note on approximating fixed points of nonexpansive mappings by the Ishikawa iteration process, J. Math. Anal. Appl. 226 (1998), no. 1, 245-250. MR 99g:47138. Zbl 916.47047.

Jialin Huang: Department of Mathematics, Yibin Teacher's College, Yibin 644007, CHINA

E-mail address: $\mathrm{j} 1$ huang@btamai 1 . net.cn 


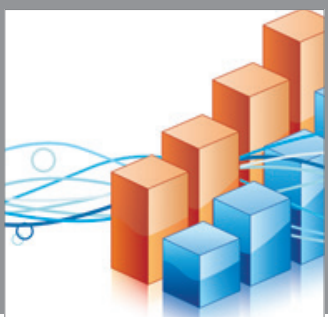

Advances in

Operations Research

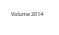

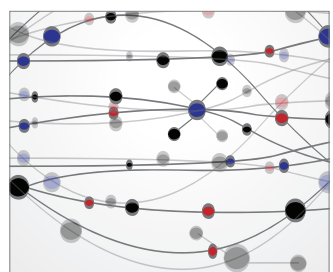

\section{The Scientific} World Journal
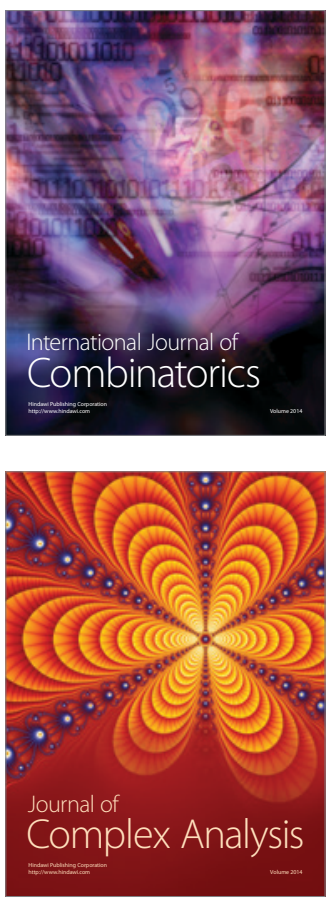

International Journal of

Mathematics and

Mathematical

Sciences
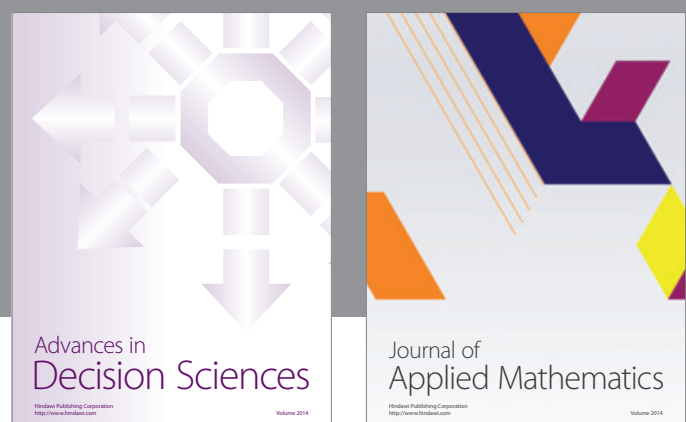

Journal of

Applied Mathematics
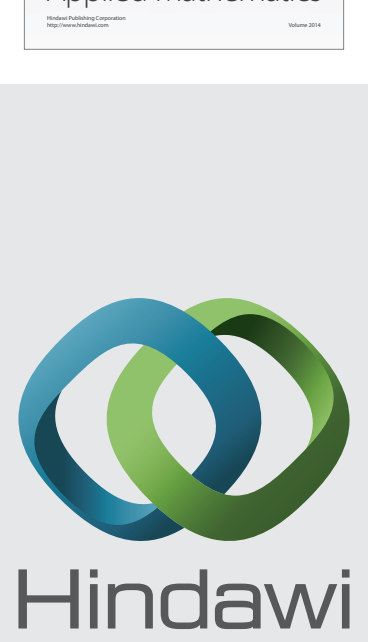

Submit your manuscripts at http://www.hindawi.com
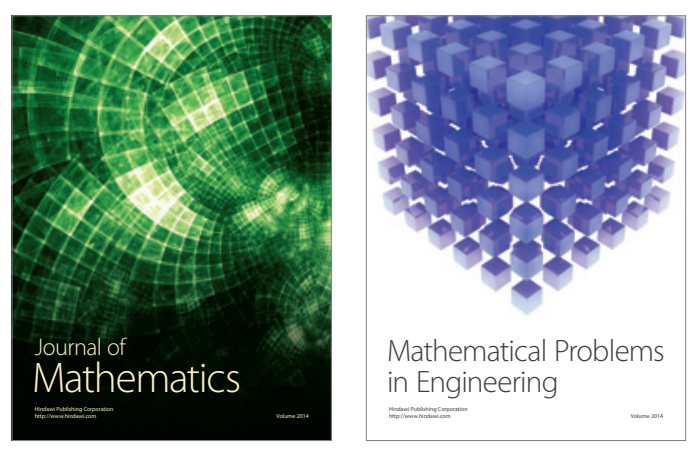

Mathematical Problems in Engineering
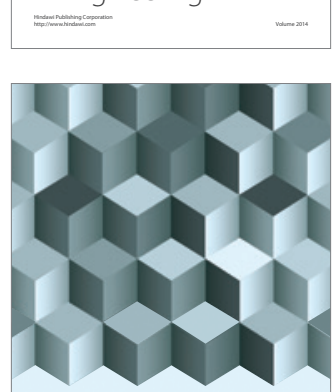

Journal of

Function Spaces
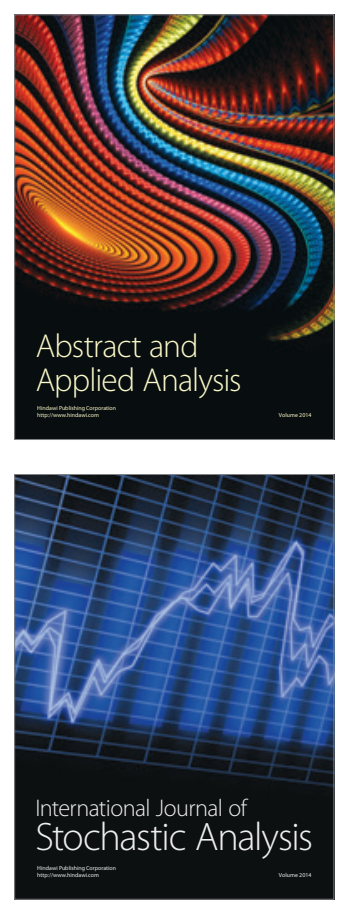

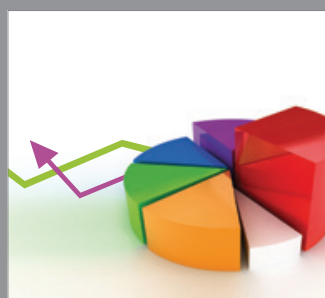

ournal of

Probability and Statistics

Promensencen
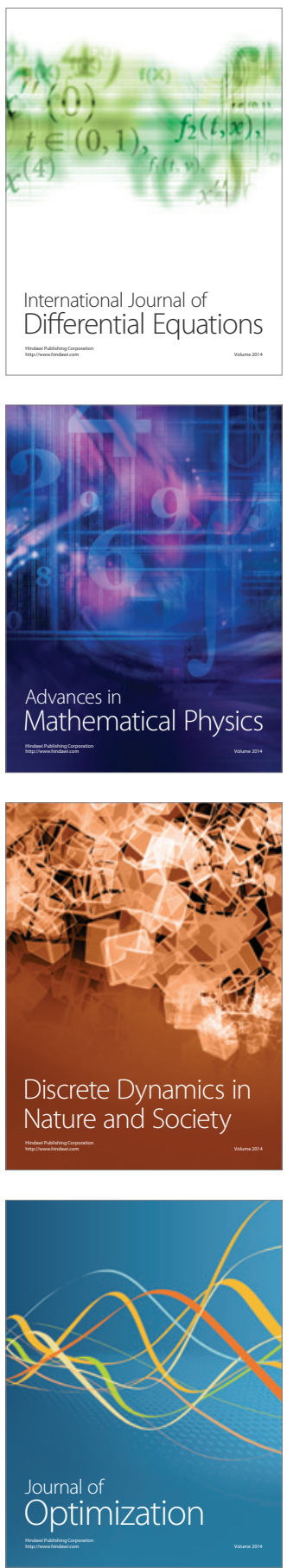